\title{
6
}

\section{The Road Ahead: A Policy Agenda for the Third Sector in Europe}

\author{
Bernard Enjolras
}

As the previous chapters made clear, the third sector in Europe is under the strain of various forces-individualization, bureaucratization and marketization—endangering third sector organizations' (TSOs') distinctive features and sustainability. The third sector has the third largest workforce in Europe and yet is invisible and lacks recognition as an economic and social force and as an interlocutor in policymaking at the European level. Our research shows that changes in the social, economic and political environment in which TSOs operate-mostly due to increased financial pressures and the introduction of market-based financing and regulatory mechanisms - are jeopardizing the sustainability of the third sector in Europe and, more importantly, increasingly undermining the distinctiveness and civic character of TSOs. Our comparative research has also uncovered innovative strategies of resilience that have enabled TSOs to tackle and cope with these challenges, though often

\footnotetext{
B. Enjolras ( $\bowtie)$

Institute for Social Research (ISF), Oslo, Norway

e-mail: bernard.enjolras@socialresearch.no

(C) The Author(s) 2018

B. Enjolras et al., The Third Sector As A Renewable Resource for Europe, https://doi.org/10.1007/978-3-319-71473-8_6
} 
with consequences that threaten the distinctiveness that has long made the third sector a positive force on the continent.

The market and bureaucratic impulses appear to be gaining the upper hand across Europe-and significantly displacing the voluntaristic and civic ones that have historically characterized this sector. Although there are significant differences across countries and civil society models depending on their institutional arrangements and public policies, this developmental trend is evident to some extent everywhere and is of importance since it puts TSOs under a survival watch, privileging adaptation strategies that pay primary attention to what has to be done in order to survive. Survival strategies may be detrimental to these organizations' distinctiveness and civic character, that is, what makes TSOs special. To be sure, these two orientations, survival and distinctiveness, are not necessarily in conflict, as illustrated by innovative resilience strategies developed by TSOs that have managed to adapt to a more competitive and marketoriented environment while retaining their distinctiveness and civic orientation. Whether such innovative resilience strategies will prevail is, to a large extent, dependent on the political environment of the third sector. ${ }^{1}$

\section{Three Scenarios}

Given the tendencies and the changes characterizing the environment of TSOs in Europe, how should the sector and its stakeholders respond and what supports will be needed? Fundamentally, three main scenarios are possible.

In the first scenario - the return to the golden age scenario-TSOs would prioritize the imperative of maintaining their distinctiveness and civic character when facing financial pressures and the increased marketization of the fields in which they operate. They would turn their backs on the government funding that is pulling them away from their distinctive character and rely more heavily on volunteering and philanthropic giving to sustain and protect their civic orientation. However, TSOs may lose

\footnotetext{
${ }^{1}$ This paragraph, and the section that follows, draw heavily on a similar diagnostic set of scenarios outlined in Lester M. Salamon, The Resilient Sector Revisited: The New Challenge to Nonprofit America. Washington: Brookings Institution Press, 2015.
} 
ground and be increasingly out-performed by for-profit actors delivering public services in a market-based regulatory environment.

In a second scenario-the drift or commercialization of the third sector scenario-TSOs would prioritize the survival imperative, adapting to their increasingly marketized environment, even if this costs losing their distinctiveness and civic character. They may rely increasingly on market resources and redesign their activities in order to serve the profitable part of the market and secure public procurement contracts in competition with for-profit actors. By doing so, they may become increasingly isomorphic with their for-profit competitors and abandon their missioncritical functions, acting as any other commercial actors.

In a third scenario, the civic economy scenario, TSOs would adapt to their transformed environment without losing their distinctiveness. They would strive to balance the imperatives of survival and distinctiveness by finding innovative solutions. In this scenario, TSOs would rethink and redesign their roles and activities in light of the economic and institutional transformations of their environment, sustaining their economic sustainability without sacrificing their mission orientation.

\section{The Civic Economy Strategy: A Policy Agenda for Europe}

Which of these scenarios will prevail depends to a great extent on the nature of the policy environment created by the EU, the national governments and the regional and local authorities in each European country. A strategy for fostering the development of a civic economy that is adapted to the realities of public finances in Europe and preserves the distinctiveness and socioeconomic contribution of the third sector in Europe needs to act on the following dimensions.

\subsection{Improving the Legitimacy and Visibility of the Third Sector in Europe}

Despite the third sector's remarkable contribution to the well-being of European citizens in terms of social service provision, opportunities and 
space for leisure activities, and despite the sector's voice function and its significant importance as a transmitter of interests and needs of the citizens, the third sector in Europe is still an unknown entity, almost invisible in the national statistics and hardly acknowledged by Eurostat. What is needed throughout Europe and in Brussels is to get the third sector out of the shadows and into the limelight of public awareness. TSOs and the sector have to be appreciated as a vital element of Europe's cultural heritage and diversity. Also, the sector's impact as a growing economic force outdistancing most major European industries in the scale of its workforce has finally to be appreciated. And the sector's political and communicative potentials, its voice function as well as its capacity to serve as an activator and incubator of citizens' engagement and volunteering, have to finally to be put up front.

However, how is this to be accomplished? How is the awareness for the achievements and potentials of the sector and its organizations to be increased? We put forward the following policy recommendations with the aim of structurally enhancing the visibility of the third sector and its organizations all over Europe and in Brussels.

\subsubsection{Improvement of the Sector's Knowledge Base}

As a crucial first step, a solid database of European third sector activity is necessary for assessing the societal, economic and political impact of the sector within the European Union. There is a decisive lack of solid statistical information on the sector and its organizations at the European level and throughout Europe. In some countries, there has been significant progress in this respect over the last decades. However, there is no concerted action across Europe. Fortunately, the United Nations Statistics Division, which oversees the System of National Accounts, the guidance system for economic data gathering around the world, has just completed work on a new handbook designed to stimulate precisely this-a comprehensive, official body of comparative statistical data on the third sector as conceptualized by this project, embracing carefully defined in-scope nonprofit organizations, cooperatives, mutuals, social enterprises and volunteer activities. We need a European task force working with the aim 
of convincing the national statistical offices and Eurostat to implement this Satellite Account on Nonprofit and Related Institutions and Volunteer Work on a priority basis to bring the European third sector out of the shadows. And we need the European Commission to provide the resources needed to underwrite the costs of the initial integration of this handbook into the statistical systems of member states. Furthermore, there are some areas of TSO activity that are worse off than others. One of these is the area of arts and culture that is also closely linked to identity building and hence to the spirit or soul of Europe. As the results of the third sector impact (TSI) project reveal, research on the impact of the third sector and its organizations is still in its infancy, and some areas of third sector activity, most prominently arts and culture, are thoroughly neglected and overlooked. To make the impact of the sector visible, the European Community has to invest in research and data gathering with a focus on third sector activities. It might be advisable for the European Commission to establish a Third Sector Research Fund to finance a robust program of research as a follow-up to the Third Sector Impact Project.

\subsubsection{Enhance Visibility through European Statutes for TSOs}

European legal stipulations for third sector activities would significantly contribute to the growth of the sector in Europe. Currently, there is not a commonly shared understanding of the third sector in Europe. In some countries, reflecting the Mediterranean and French social economy tradition, third sector activities are perceived as being commensurate with business endeavors; in other European regions, the third sector is looked upon as a synonym for voluntary activity based on the principle of reciprocity. Still today, legal stipulations strongly reflect the regional traditions and legacies of history of the various European countries. But diversity and variation with respect to the legal forms definitely stand in the way of the development of a European third sector identity. In addition, the variety of legal forms translates into a significant hurdle for data gathering. Finally, umbrellas of TSOs working in Brussels are faced with significant difficulties in speaking with just one voice when they stand for legally very different constituencies. Introducing European Statutes for 
third sector service providers, voluntary associations and foundations would definitely be a big step ahead in the direction of the Europeanization of the sector.

\subsubsection{Make the Voice of the Sector Heard}

A decisive lack of infrastructural backing in terms of support organizations as well as "umbrellas" that are able to give a voice to the needs of TSOs and volunteers at the various levels of European governance, in Brussels and in the capitals of Europe, turned out to be a significant deficiency of the sector throughout Europe. There are many reasons why it is necessary to facilitate the establishment of third sector support infrastructures. In countries without a long tradition of third sector social service provision, TSOs operating in close cooperation with the government are perceived as semi-public organizations that sometimes are prone to clientelism or even corruption. Umbrellas of the sector are necessary to help TSOs in these countries escape the legitimacy gap. In countries looking back upon a long tradition of partnership with the government, it is increasingly necessary to draw attention to the distinctiveness of TSOs. These organizations are major employers; they speak to the needs of increasingly heterogeneous communities, and due to their economic strength, they are in a position to voice the negative side of neoliberal marketization. However, even the big players of the third sector need umbrellas, in particular in Brussels, in order to make their claims heard. Therefore, beyond setting up and supporting "umbrellas," stakeholders suggested establishing ombudsmen and/or ombudswomen for the third sector and its organizations inside key political institutions, such as the European and National Parliaments. Furthermore, public awareness nowadays constitutes an outcome of media coverage. Here, the sector and its organizations are significantly overlooked. There is some notice of the sector and its achievements when major humanitarian or ecological disasters happen throughout the world. However, the media thoroughly falls short in providing a realistic and simultaneously appealing picture of the sector and its organizations. Instead, the media looks out for cases of embezzlement and cases of corruption. If there is no broker in terms of 
umbrella organizations working on behalf of the sector, there is little chance that lack of awareness of the sector does not change into skepticism or even mistrust.

\subsubsection{Help TSOs to Market Themselves}

TSOs not only need "umbrellas" to make their voice heard in the wider public and in the political arena; they also need support to adapt to a significantly changed environment. They need help to modernize themselves, particularly with respect to both public relations and marketing. Although new web-based technologies carry enormous potentials, TSOs are limited in their capacity to adapt to technological change. Particularly small- and medium-sized TSOs embedded in local communities and working primarily with volunteers face difficulties in addressing the public with the help of new technology. They are, as our stakeholders indicate, simply not accustomed and also not well equipped to address all these new publics that are of increasing importance for both the visibility of the organization and the new modes of acquiring resources or organizing constituencies. For sure, civic actions are increasingly organized on the net (e.g. flash mobs); with the support of the net, citizens also increasingly arrange ad-hoc sporting activities; additionally, campaigns are progressively formed within social media, and volunteers gather on social platforms for joint community action. The success story of crowd funding was primarily made possible by the World Wide Web. Furthermore, many of the activities organized via the web take place outside formal organizations. This trend might result in a situation in which TSOs are losing relevance to European citizens. Doubtlessly, modern communication technology is providing new ways of organizing support and of informing and empowering people and therefore constitutes a valuable asset for the third sector. Consequently, TSOs should increasingly make use of technological and communication tools in order to develop their potential as social impact generators. However, the technological knowhow of TSOs is by and large rather limited, primarily used by larger TSOs and furthermore restrained by either the nonexistence of or limited access to TSO-support organizations. The need for help and assistance is 
particularly prominent in areas of TSO activity beyond social service provision, such as the arts and culture. There is urgency for building up infrastructural support organizations and intermediaries to facilitate access to modern communication technology. Philanthropy, but also public funders, should invest in infrastructural support organizations that address the needs of TSOs active in certain fields and areas. This could serve as a structural backbone for those new demands that come with technological change.

\subsubsection{Get the Public Back In-Provide Space for Third Sector Activities}

Small TSOs engaged in sports, arts and culture or other communitybased areas are increasingly facing the difficulty of finding "space" and facilities for their activities all over Europe. In metropolitan areas with rising shortages of residential space, it also has become increasingly difficult for TSOs that are active in interest representation to find places for encounters and gatherings. Due to gentrification, areas and locations that used to be public spaces are converted into upscale real estate or commercial centers. In municipalities under austerity regimes, public facilities such as gyms, auditoriums, exhibitions or concert halls are often in very poor condition and in urgent need of renovation. Since municipalities have to make money, renting these spaces has turned into a costly affair. On the other hand, the majority of small and middle-sized TSOs are not able to afford operating their own venues and facilities. Consequently, compared with commercial providers, many TSOs, in particular, sport clubs, but also those who are active in the arts and culture, have lost attractiveness, and they are limited in pursuing their specific activities. This has a decisive effect on local community life, as arts and culture, as well as sports, are the most popular areas of volunteer commitment; the venues of these TSOs serve as points of crystallization for social life in neighborhoods. Local governments should be aware that there is an urgent need for public spaces and facilities in order to enrich and sustain community life. Simultaneously, national governments and the European Union should finally recognize that the provision and 
maintenance of public facilities and public spaces constitute a solid investment into the future of Europe. And finally, TSOs could also help get the public back by pooling their resources and cooperating in acquiring and maintaining facilities.

\subsection{Improving Third Sector Finances and Government-Third Sector Partnership}

In addition to improving TSO legitimacy and visibility, a second broad set of strategies is needed to address third sector finances and governmentthird sector partnerships. All over Europe, TSOs are struggling to make ends meet. The reasons why TSOs are facing a hard time are manifold. As the results of the TSI project indicate, acquisition of resources, finances and personnel, has changed significantly for TSOs during the last decades. Partly, this constitutes an outcome of modified TSO-government relations caused by the neoliberal-inspired introduction of competitive markets for social services and instruments of new public management (NPM), such as competitive tendering or contract management. But other factors are also at work, and several steps will be needed to address this strategic priority.

\subsubsection{Get Structural Funds Back In}

In the first place, there is an urgent need for structural funds all over Europe in order to safeguard the durability and sustainability of both TSOs and government-third sector cooperation. All over Europe, public funding nowadays is by and large based on temporary contracts. Furthermore, governments have generally shifted from so-called institutional or structural funding to temporary funding related to specified projects. Therefore, TSOs that bounce from one project to another have less and less capacity for building up and maintaining an organizational infrastructure that safeguards stability and allows quick responses to new challenges. The need for more sustainable funding is very acute in those regions where TSOs are not accepted partners of public service delivery, such as in Eastern Europe and the Mediterranean region. Furthermore, 
TSOs that are active in leisure and community-oriented areas such as arts and culture or sports, where the sector consists of myriads of small organizations, small structural funds would significantly contribute to a more sustainable development of the sector. European foundations also have a role to play in providing such core support. There is no way that those TSOs can exclusively live on so-called project money. They are in need of long-term structural public and private support that constitutes a bottom-up approach and an investment into sustainable community development.

\subsubsection{Lift up the Burden of Procurement Procedures}

The introduction of NPM has had a decisive impact on third sectorgovernment relations, and particularly on the modes of financing. Funding for TSOs is increasingly acquired through competitive grants and contracts. As a result of the new funding environment, third sector bureaucratization has developed into a major obstacle impacting negatively on TSOs. The accountability requirements accompanying procurement have increased significantly during the last decades all over Europe. This translates into a situation in which TSOs have to allocate more and more resources to complying with the administrative requirements of applying, reporting and evaluating grants and contracts instead of devoting time and energy for "doing the right thing" and supporting the community. Urgently needed at every level of governance in Europe are new modes of procurement that take the specificity of TSOs into account. Again, TSOs are not proxies for commercial enterprises. Modes of procurement have to be reevaluated and ideally homogenized throughout Europe. They should also incorporate a so-called social clause-giving priority in public procurement to TSOs having a social impact-that will ease competition between TSOs and for-profits; they should provide modes for cooperation between governments and TSOs that are tailored toward the needs of TSOs; they should specify tasks and functions for both sides in order to enhance cooperation instead of creating a culture of surveillance; and finally, without losing attention to accountability, they should simplify monitoring and evaluation procedures. The need to 
reduce the complexity of procurement procedures is especially necessary at the European level. EU-procurement and funding procedures are far too complicated for the majority of European TSOs. There is a decisive need to adapt the European directive on public procurement to the needs of TSOs and, in particular, to those active in interest representation and lobbying. In addition, the European directive on VAT needs to be altered. A more favorable tax framework has to be introduced that envisages a reduction of VAT for TSOs and that makes tax reimbursement for TSOs possible. Moreover, the policies of matching funds for EU-grants have to be changed. It should be made possible to use specific third sector resources, for example, voluntary work, as an equivalent to money, in order to pave the way for smaller organizations to get access to EU-support.

\subsubsection{Facilitate Access to Capital Markets and to Alternative Sources of Financing}

As the results of the TSI project clearly show, public money is no longer the prime source of income for TSOs in several fields of activities. Despite increasingly competitive environments, the economic success story of the sector has been impressive in Europe. Nevertheless, TSOs are not yet treated on an equal footing with for-profit enterprises. This is particularly the case with respect to their access to capital markets. Banks and other financing institutions are very reluctant to do business with TSOs. Additionally, most TSOs cannot access equity finance due to their nonprofit distribution constraint and legal inability to share ownership with shareholders. A change of culture is needed as well as an empowerment strategy for TSOs supporting them in getting access to capital markets and in experimenting with new modes of financing such as crowd funding, social loans and cooperation with ethical banks. ${ }^{2}$

\footnotetext{
${ }^{2}$ For a discussion of how this can be done, see Lester M. Salamon, Leverage for Good: An Introduction to the New Frontiers of Philanthropy and Social Investment. New York: Oxford University Press, 2014a; and Lester M. Salamon, ed. The New Frontiers of Philanthropy: A Guide to the New Actos and Tools Reshaping Global Philanthropy and Social Investment. Oxford: Oxford University Press, 2014b.
} 


\section{$3 \quad$ Fostering Foundations' Supportive Role of the Third Sector in Europe}

Philanthropic institutions and especially foundations have the potential to become the backbone of the third sector; they should play a critical role in canalizing private funds to value-oriented projects emanating from third sector initiatives and as supporters of the entire European third sector by helping building up its visibility and capacity. As of now, most foundations in Europe assume no responsibility for the sector as a whole. Yet, they are the only source of alternative funding (to public funding) for these sector-wide objectives.

Because foundations have limited resources, they need to act strategically orienting the use of their limited funds to activities to maximize leverage and impact. Paul Brest and Hal Harvey capture the basic issues and motivation of strategic philanthropy: "Accomplishing philanthropic goals requires having great clarity about what those goals are and specifying indicators of success before beginning a philanthropic project. (...) This, in turn, requires an empirical, evidence-based understanding of the external world in which the plan will operate" (Brest and Harvey 2008: 7). Supporting the third sector as a whole by helping TSOs to build their capacity constitutes a prioritized avenue for European foundations to maximize their impact. By helping build the capacity of the third sector as a whole, they will generate broader socioeconomic impacts than by just focusing on particular projects.

\section{Improving the Attractiveness of TSOs}

A third broad strategy needed to sustain the core capabilities and values of the third sector in Europe is to improve the overall attractiveness of TSOs to their fundamental constituencies. The new culture of volunteering, which is characterized by a high degree of volatility, significantly affects membership affiliation and the concept of the voluntary association that used to be the nucleus and core concept of European TSOs. Indeed, there is a real danger that the third sector in its current contours and how it has developed in Europe over the years will not be capable of continuing to be a thriving societal force in Europe that gives people a voice, provides opportunities for community and leisure activities and, last but not least, consti- 
tutes an important provider of social services, contributing to the community life and well-being of European citizens. The environment of TSOs needs to be enhanced in order to increase the attractiveness of these organizations for volunteers and paid staff. However, TSOs need to improve awareness of their values and distinctive features.

\subsection{Renewing the Third Sector's Values}

At the center of the civic economy scenario must be a clarification of the third sector's values, the distinctive qualities and attributes that TSOs bring to society in Europe. The third sector's distinctive contribution is closely related to a set of values such as equity, openness, empowerment, participation, responsiveness and commitment to the enrichment of human life. The marketization of the sector, accompanied by an emphasis on metrics that privileges the service function of TSOs, contributes to the downplaying of these values. Making these values visible and clearly articulated for the sector as a whole and for particular organizations will be crucial for the success of the civic economy renewal of the third sector and the sustainability of its specific contribution to society in Europe.

\subsection{Increase the Attractiveness of TSOs as Employers}

Despite high qualifications and outstanding commitment, TSOemployees are faced with deteriorating working conditions. Low salaries, which are not comparable with those paid in the commercial sector, constitute a further outcome of the changed environment and funding situation of TSOs in several parts of the third sector. Consequently, working in the third sector becomes increasingly unattractive. Particularly in areas where the mental and physical stress is high, TSOs face serious problems to recruit highly qualified personnel. Hence, it is most likely that in the near future the quality of services will deteriorate as TSOs have to employ less professional personnel and reduce personnel costs per service unit. Women are particularly hit hard by the degraded working conditions in the sector, as they account for the largest part of the sector's labor force. Introduction of collective wage agreements in prominent areas of the 
sector, for example, social services, provide an effective tool for stopping the ruinous competition for contracts. TSOs are called upon to agree with their employees on fair labor standards throughout the sector. In addition, the specificity of third sector management and governance has hardly been recognized by institutions of higher education throughout Europe. Our stakeholders time and again indicated that there is a decisive lack of training facilities and courses tailored to the needs of third sector managers who have to get along with a broad spectrum of very different constituencies and who also should never lose track of the mission and vision of the respective TSO.

\subsection{Increase the Attractiveness of TSOs for Volunteers}

Volunteers are a pivotal source of legitimacy, community orientation and embeddedness for TSOs. However, volunteer engagement is not free. All over Europe, there is a trend for more flexible, fluid and tailor-made forms of volunteering. Life-long commitment to a specific TSO, as used to be the case in former times, no longer reflects the reality of volunteering in Europe today. The modernized culture of volunteering is also characterized by a new expressiveness of volunteers. They have become more vocal about their voluntary commitment. Nowadays, volunteers want to decide how they become active, how long they volunteer and where they specifically get engaged. This new voluntarism forces TSOs to install procedures of continuing infrastructural support, indeed, volunteer management. It also requires further training of volunteers as well as of the TSO-personnel who are in charge of coordinating and monitoring volunteer work.

\subsection{Increase the Attractiveness of TSOs for Honorary Board Members}

A dual governance structure that encompasses honorary board members or trustees and full-time professional managers still seems to be an appropriate governance model for many TSOs in Europe. However, as indicated by our research and by many of our stakeholders, there are escalating problems around recruitment for governance roles, particularly with respect to the 
honorary positions of board members. Again, the reasons for this development are manifold and closely related to the increased responsibilities of board members, which are partly a result of the changed procurement procedures. In addition, in local communities, there nowadays are many opportunities to get involved and to serve in prestigious positions without being responsible for hundreds of employees or millions of Euros. In order to safeguard the local embeddedness of TSOs through community trusteeship and honorary board members, those who are willing to be engaged have to be supported and provided with task- oriented training programs and courses that indeed speak to their needs.

\section{Conclusion}

In a time when Europe faces economic and social developments characterized by accelerated technological changes, increased globalization of world markets, increased migration flows and a shift in the economic opportunity structure creating rising inequalities among individuals and national communities, the collective capacity embedded in the third sector constitutes a major factor of social resilience (Hall and Lamont 2013) of Europe.

Indeed, when facing the challenges posed by rapid socioeconomic transformations, entailing economic reorganization, social dislocation and redistributive effects, the capacity of groups and communities to adapt to transformations and to secure their well-being in spite of these transformations - their degree of resilience-is dependent upon their collective capacities. Individuals' social resilience, their capacity to mobilize resources and solidarities, is enhanced by social organization. TSOs and the third sector in its entirety, in interplay with the state, play a crucial role in enhancing social resilience by fostering social solidarities, social capital, capacities for collective action, and the provision of collective goods.

The third sector and volunteering truly represent a unique "renewable resource for social and environmental problem-solving" for Europe. The results of our research have provided evidence supporting this claim. They have also shown that the third sector in Europe is subject to major changes and challenges. The future developmental path of Europe 
depends not only on its economy and polity, but also on the dynamism of its third sector. Policy adjustments are needed in order to support a dynamic third sector in Europe and preserve the distinctiveness of the organizations that compose it. However, these policy adjustments will not take place without a better public understanding of the state of the third sector in Europe. Hopefully, the analysis developed in this report will contribute to such an understanding.

\section{References}

Brest, P., \& Harvey, H. (2008). Money well spent: A strategic plan for smart philanthropy. New York: Bloomberg Press.

Hall, P. A., \& Lamont, M. (2013). Social resilience in the neoliberal era. New York: Cambridge University Press.

Salamon, L. M. (2014a). Leverage for good: An introduction to the new frontiers of philanthropy and social investment. New York: Oxford University Press.

Salamon, L. M. (2014b). The new frontiers of philanthropy: A guide to the new actos and tools reshaping global philanthropy and social investment. Oxford: Oxford University Press.

Salamon, L. M. (2015). The resilient sector revisited: The future of nonprofit America (2nd ed.). Washington, DC: Brookings Institution.

Open Access This chapter is distributed under the terms of the Creative Commons Attribution 4.0 International License (http://creativecommons.org/ licenses/by/4.0/), which permits use, duplication, adaptation, distribution and reproduction in any medium or format, as long as you give appropriate credit to the original author(s) and the source, a link is provided to the Creative Commons license and any changes made are indicated.

The images or other third party material in this chapter are included in the work's Creative Commons license, unless indicated otherwise in the credit line; if such material is not included in the work's Creative Commons license and the respective action is not permitted by statutory regulation, users will need to obtain permission from the license holder to duplicate, adapt or reproduce the material. 